\title{
INFLUENCE OF LOADING RATE ON SHEAR FRACTURE TOUGHNESS FOR FAILURE MODE TRANSITION
}

\author{
J.F. Kalthoff, A. Bürgel \\ Experimental Mechanics \\ Ruhr-University Bochum, Germany
}

\begin{abstract}
When subjected to shear loading of sufficiently high rate, many materials do not fail by cracks, but by adiabatic shear bands. Work is reported on investigations for determining the dependence of the impact shear fracture toughness as a function of loading rate, in particular in the regime of failure mode transition from cracks to adiabatic shear bands. For achieving high rate shear loading, edge cracked specimens are asymmetrically impacted at the cracked edge by a projectile accelerated by an air gun to velocities up to 100 $\mathrm{m} / \mathrm{s}$. The resulting mode-II stress intensity factors and the times of onset of failure are determined by a specially developed strain gauge measuring technique. Results on shear fracture toughnesses with increasing loading rate are reported for two structural materials, a 1\% chromium steel and a high strength aluminum alloy. Whereas decreasing fracture toughnesses are observed with increasing loading rate when failure occurs by tensile cracks, the fracture toughness increases with loading rate when failure occurs by adiabatic shear bands.
\end{abstract}

\section{KEYWORDS}

Fracture toughness, shear loading, rate dependence, adiabatic shear band, failure mode transition

\section{INTRODUCTION}

In the regime of linear elastic or small scale yielding fracture mechanics, cracks that are subjected to quasistatic shear mode-II conditions of loading fail by the initiation of tensile mode-I cracks, propagating of an angle of about $70^{\circ}$ with respect to the ligament. When the loading is applied dynamically, e.g. by an impact event, a failure mode transition $[1,2]$ is observed when the loading rate exceeds a certain limit value: failure then occurs by an adiabatic shear band propagating almost in the direction of the ligament. These adiabatic shear bands absorb more energy for propagation than cracks. This paper is aimed to investigate the impact shear mode-II fracture toughness $\mathrm{K}_{\text {IId }}$ as a function of loading rate, in particular in the regime of failure mode transition where the energy dissipation changes from a low to a high energy process [3].

\section{EXPERIMENTAL TECHNIQUES}

For achieving high rate shear conditions of loading, edge cracked specimens are asymmetrically impacted at the cracked edge by a projectile accelerated by an air gun to velocities up to $100 \mathrm{~m} / \mathrm{s}$ (LECEI-technique, see Fig. 1). A compressive wave is initiated to one side of the crack, resulting in a shear-(mode-II)-loading after 
the wave has reached the crack tip. The mode-II loading condition gets disturbed after interference of waves reflected at the boundary of the specimen with the crack tip stress distribution. Details of the LECEItechnique are reported in [2].

For determining mode-II stress intensity factors during the phase of loading, a strain gauge measuring technique has been developed [3,4] which is based on a concept introduced by Dally, Sanford [5] for the mode-I case. A strain gauge is mounted on the specimen near the crack tip. The angular position of the strain gauge is specially chosen to eliminate or to minimize the influence of two of the higher order terms of the crack tip stress field. The stress intensity factor is then determined from the measured strain. Two strain gauges are used for extrapolating the measured data against the actual crack tip position. In addition, static precalibration of the strain gauges is applied. Instability, i.e. initiation of failure is indicated by a change of the slope of the strain gauge signal as a function of time: In the case of failure by a crack (which propagates at an angle of about $70^{\circ}$ with respect to the ligament - in a downward direction in Fig. 1), the distance between the crack tip and the strain gauge increases and, consequently, the strain gauge signal decreases; in the case of failure by an adiabatic shear band (which propagates almost in the direction of the ligament, i.e. towards the strain gauge), the distance from the failure tip to the strain gauge is decreased and the signal increases. Additional information on the failure event is obtained by direct high speed recordings taken with a Cranz-Schardin 24 spark high speed camera. The stress intensity factor $\mathrm{K}_{\mathrm{II}}$ derived from the measured strain gauge signal at the moment of instability, i.e. at the time of onset of failure by a crack or by an adiabatic shear band, defines the impact mode-II fracture toughness $\mathrm{K}_{\text {IId. }}^{*}$.

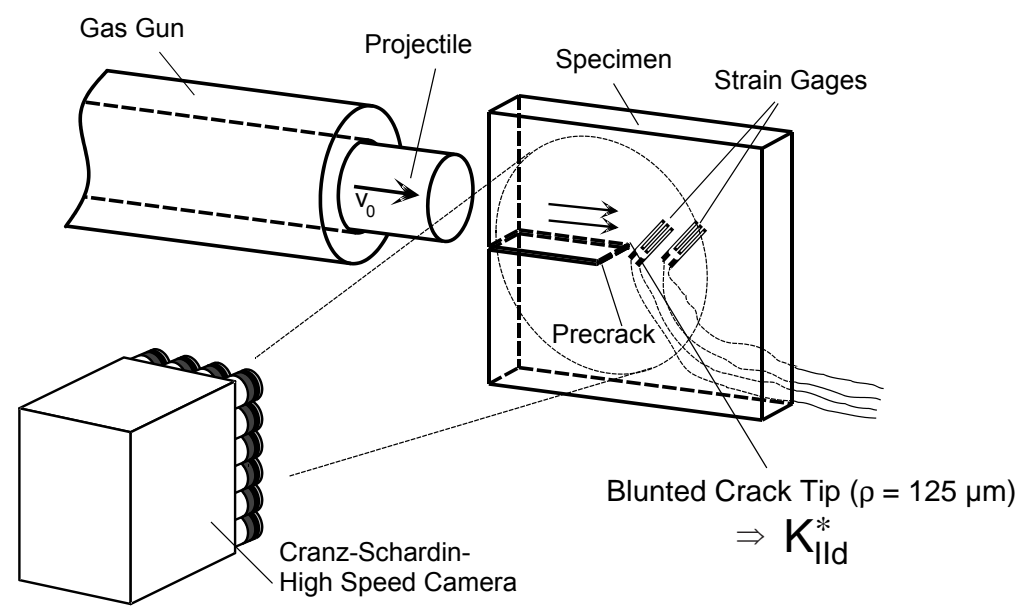

Figure 1: Experimental set-up (schematically)

Two materials are investigated, a $1 \%$ chromium steel and a high strength aluminum alloy. The specimen size used for both series of investigations is $150 \mathrm{~mm}$ x $150 \mathrm{~mm}$, the thickness of the specimens is $20 \mathrm{~mm}$. The specimens are prepared with initial cracks/notches of about $60 \mathrm{~mm}$ length oriented in TL direction. At its tip the notch/crack is extended by a few $\mathrm{mm}$ by a special saw resulting in a finite bluntness at the tip of the crack. The determined fracture toughnesses, thus, are not true fracture toughness values that apply for a sharp pre-crack, but finite bluntness fracture toughnesses are measured, characterized by the symbol $\mathrm{K}_{\text {IId. }}^{*}$

\section{SHEAR FRACTURE TOUGHNESSES}

The $1 \%$ chromium steel 42 CrMo 4 (German designation) has been investigated. The steel was heat treated at $860{ }^{\circ} \mathrm{C}(2 \mathrm{~h})$, quenched (oil) and tempered at $160{ }^{\circ} \mathrm{C}(3 \mathrm{~h})$. In its final condition the steel showed a yield strength $\sigma_{\mathrm{Y}}$ of about $1570 \mathrm{MPa}$ and a tensile mode-I fracture toughness $\mathrm{K}_{\mathrm{Ic}}^{*}(\rho=125 \mu \mathrm{m})$ of about 100 $\mathrm{MN} / \mathrm{m}^{3 / 2}$.

Series of experiments have been performed with systematic variations of the impact velocity ranging from $20 \mathrm{~m} / \mathrm{s}$ to $90 \mathrm{~m} / \mathrm{s}$. Typical results are given in Fig. 2. Failure paths and failure surfaces are shown on the left hand side of the figure for a low rate experiment $(30 \mathrm{~m} / \mathrm{s}$ impact velocity) and on the right hand side of the figure for a high rate experiment $(60 \mathrm{~m} / \mathrm{s}$ impact velocity). In the low rate experiment, failure occurs by a tensile crack propagating at an angle of about $-70^{\circ}$ with respect to the ligament (for the beginning of the 
failure path); the failure surface is a typical fracture surface with roughness (plus additional indications of delamination in the middle of the specimen) and shear lips at the edges of the specimen, although small in size because of the high yield strength of the material. In the high rate experiment, failure due to an adiabatic shear band is observed propagating almost straight in the direction of the ligament (more precisely in the direction of few degrees to the opposite side of the ligament tensile cracks would propagate). The adiabatic shear band propagates over a considerable length (about $30 \mathrm{~mm}$ ) and then is arrested. The length of the shear band at the edges of the specimen in all cases is larger than in the middle of the specimen, a behaviour opposite to what is observed for cracks. The failure following the adiabatic shear band is due to late time effects, e.g. due to arbitrary impact events in the catcher tank, when the specimen is decelerated. The adiabatic shear band has a typical shiny, mirror-like appearance, shear lips cannot be identified.
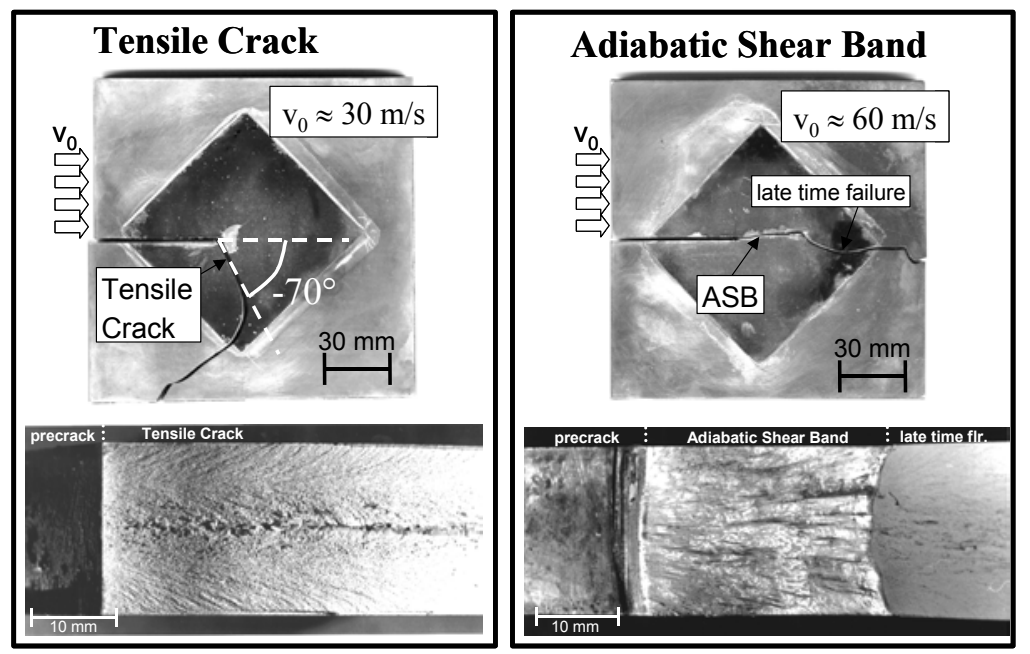

Figure 2: Failure in steel 42 CrMo 4 by tensile cracks or adiabatic shear bands in the low or high rate regime

Figure 3 gives typical strain gauge signals with the strains converted to stress intensity factors obtained in a low and a high rate test. The signals of both strain gauges SG1 and SG2 are shown. In the low rate experiment, an abrupt change of the signal to a decreasing slope is observed, indicating failure by a tensile crack. In the high rate experiment, on the other hand, the slope of the strain gauge signal from a certain time on gets steeper, indicating failure by an adiabatic shear band. The derived times of onset of failure agree with those obtained from high speed photographic recordings of the event.
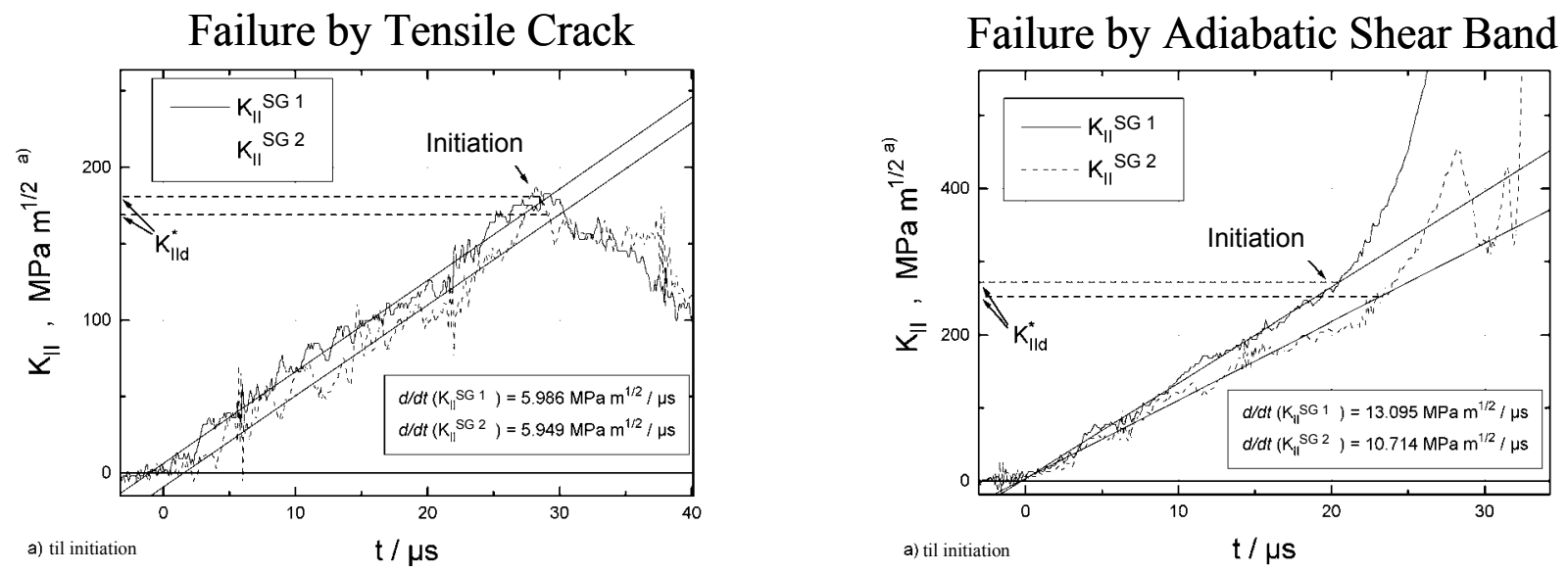

Figure 3: Strain gauge response for failure by tensile cracks and adiabatic shear bands in steel 42 CrMo 4

The shear mode-II fracture toughnesses measured as function of loading rate are shown in Fig. 4. Depending on which failure mode gets activated a different rate dependence is observed: For failure by tensile cracks which is obtained in low rate tests for loading rates $\dot{\mathrm{K}}_{\mathrm{II}}<7 \cdot 10^{6} \mathrm{MN} / \mathrm{m}^{3 / 2} \mathrm{~s}$, the shear mode-II fracture toughness $\mathrm{K}_{\text {IId }}^{*}$ decreases with loading rate, a behaviour as it is regularly observed for mode-I loading as well. 
But, for failure by adiabatic shear bands, which results in the high rate tests for loading rates $\dot{\mathrm{K}}_{\mathrm{II}}>7 \cdot 10^{6}$ $\mathrm{MN} / \mathrm{m}^{3 / 2} \mathrm{~s}$, the shear mode-II fracture toughness $\mathrm{K}_{\text {IId }}^{*}$ shows an increasing trend with loading rate. The transition between the two failure modes is not abrupt, a transition regime is observed for which the one or the other failure mode can become activated. There is no jump of the shear mode-II fracture toughness in this transition regime, the two curves overlap with their individual tendencies maintained.

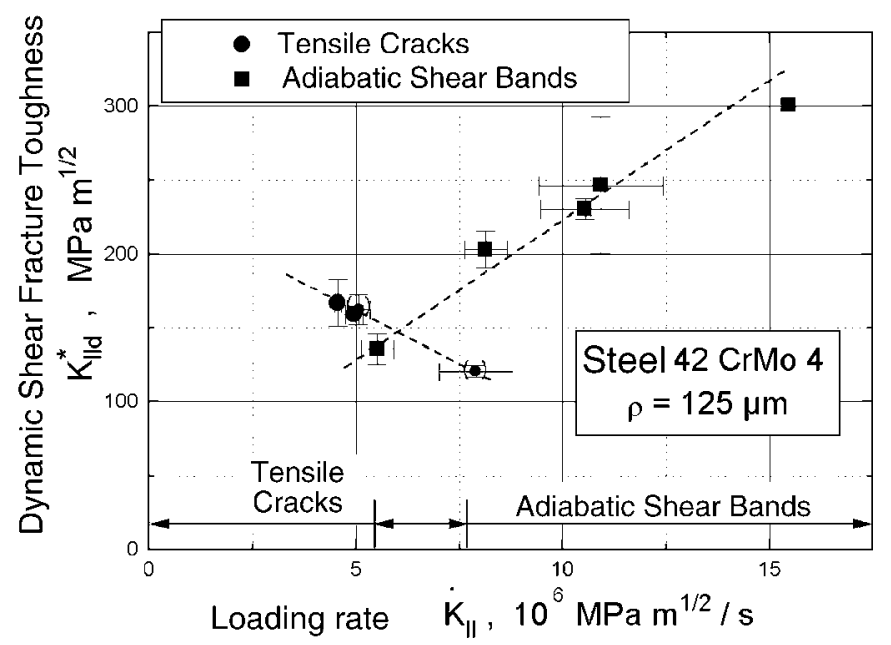

Figure 4: Dependence of shear fracture toughness from loading rate for failure mode transition in steel $42 \mathrm{CrMo} 4$

The second material investigated is the high strength aluminum alloy Al 7075. The material is used in T6 condition. The relevant material properties were measured to: yield strength $\sigma_{Y}=620 \mathrm{MPa}$, quasi-static precrack mode-I fracture toughness $\mathrm{K}_{\mathrm{Ic}} \approx 27 \mathrm{MN} / \mathrm{m}^{3 / 2}$.

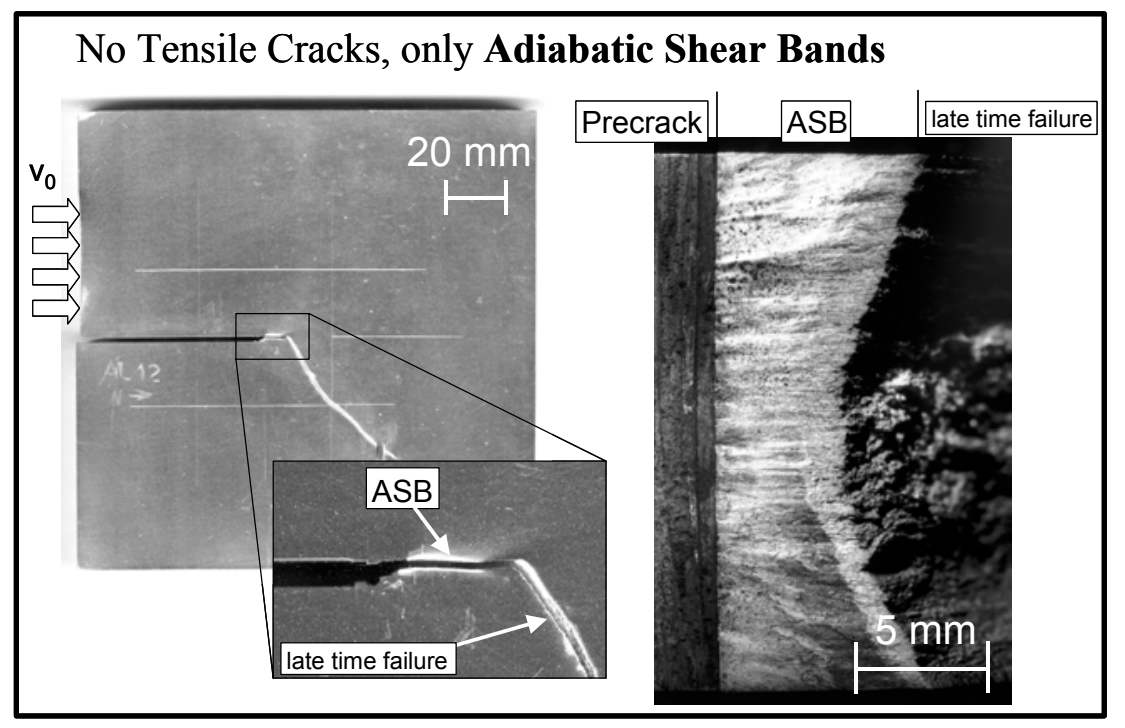

Figure 5: Failure in aluminum alloy Al 7075 by adiabatic shear bands

Results on the failure path and the failure surface are shown in Fig. 5. In all experiments failure by adiabatic shear bands resulted. Failure by tensile cracks at low loading rates could not be observed. In order to further explore this special behaviour, additional experiments have been performed at loading rates reduced down to even quasi-static conditions [6,7]. These experiments showed failure by (adiabatic) shear bands with the same or similar basic characteristics as observed in the dynamic tests. This behaviour is in contradiction to the generally accepted rule that the maximum tangential stress criterion controls the failure path in classical quasi-static linear elastic fracture mechanics. Other researchers have observed the same or a similar behaviour for mode-II or mixed mode loaded cracks in aluminum alloy, see in [7]. This unusual behaviour is not completely understood yet, it is believed that it is a peculiarity of the material aluminum. (In order not to be misunderstood: mode-I tensile crack propagation certainly does exist in aluminum alloy and is observed 
in experiments, but for mode-I loading conditions only.) The phenomena and characteristics of adiabatic shear bands observed with the aluminum alloy are the same as observed with steel: The shear bands extend almost straight in the direction of the ligament, they are typically $8 \mathrm{~mm}$ long. The length of the band is larger at the edges of the specimen than in the middle of the specimen. The adiabatic shear band has a shiny, mirror-like appearance, shear lips do not exist. Figure 6 gives typical strain gauge signals observed in the aluminum tests. The increase in the slopes of the curves indicates that failure occurred by an adiabatic shear band.

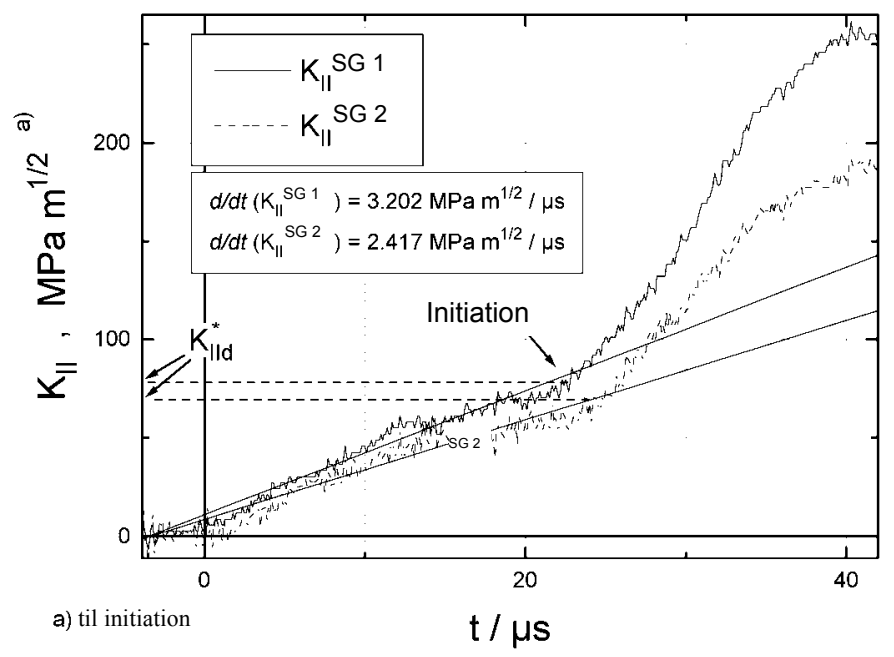

Figure 6: Strain gauge response for failure by adiabatic shear bands in aluminum alloy $\mathrm{Al} 7075$

The shear mode-II fracture toughnesses measured as function of loading rate are shown in Fig. 7. In all the tests which resulted in loading rates $\dot{\mathrm{K}}_{\mathrm{II}}>2 \cdot 10^{6} \mathrm{MN} / \mathrm{m}^{3 / 2}$, the shear mode-II fracture toughness $\mathrm{K}_{\text {IId }}^{*}$ measured for failure by adiabatic shear bands shows a strongly increasing trend with loading rate, similar as observed in the steel tests.

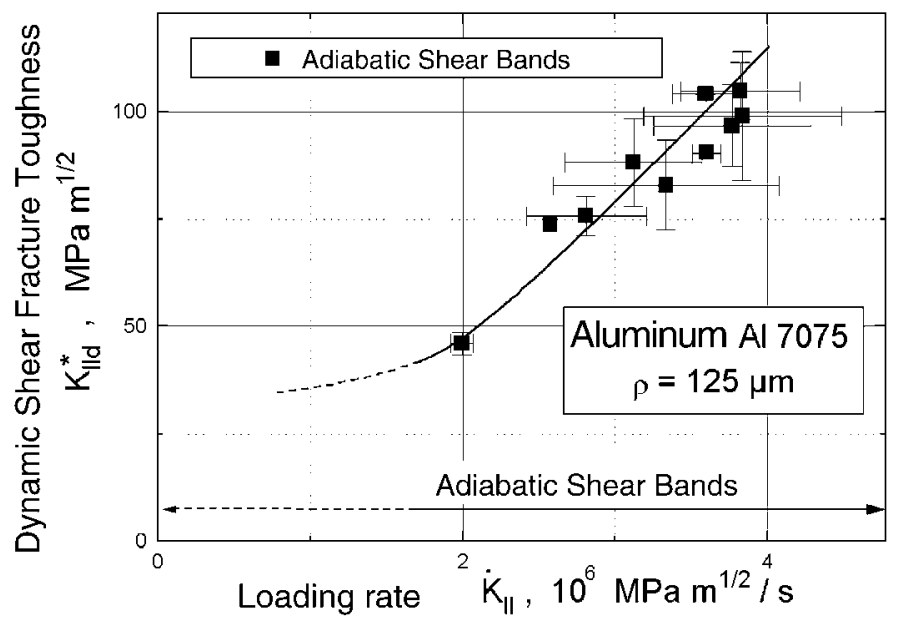

Figure 7: Dependence of shear fracture toughness from loading rate for failure by adiabatic shear bands in aluminum alloy $\mathrm{Al} 7075$

\section{SUMMARY AND DISCUSSION}

Shear mode-II fracture toughnesses $\mathrm{K}_{\text {IId }}^{*}$ are measured as a function of loading rate. The steel 42 CrMo 4 shows the following behaviour: for failure by tensile cracks at low loading rates the impact fracture toughness decreases with loading rate; for failure by adiabatic shear bands at higher loading rates the fracture toughness increases with loading rate. With the aluminum alloy Al 7075 failure by adiabatic shear bands is observed at all loading rates applied and fracture toughnesses increasing with loading rate result. 
The decreasing trend of the fracture toughness with loading rates for crack failure is certainly due to the regular strain rate hardening effects: with increasing loading rate the yield strength of the material increases, i.e. the resulting plastic zone at the crack tip and in turn the fracture toughness decreases. The increasing trend of the fracture toughness with loading rate for failure by adiabatic shear bands is speculated to be caused by thermal softening effects which are a result of the adiabatic conditions and the extreme increases in temperature that control the process of adiabatic shear band failure: yielding of the material and plasticity effects become more pronounced which dissipate more energy, and thus, result in higher fracture toughness values with increasing loading rate. The processes in detail are more complicated in nature of course. Figure 8 shows micrographs of regions underneath the failure surfaces formed by a tensile crack and by an adiabatic shear band. In the case of failure by the tensile crack, the base material with its characteristic structure extends completely up to the failure surface. In the case of failure by the adiabatic shear band, the extreme heating effects result in phase transformations of the shear band zone adjacent to the failure surface which are accompanied by an increase of the hardness of the material.
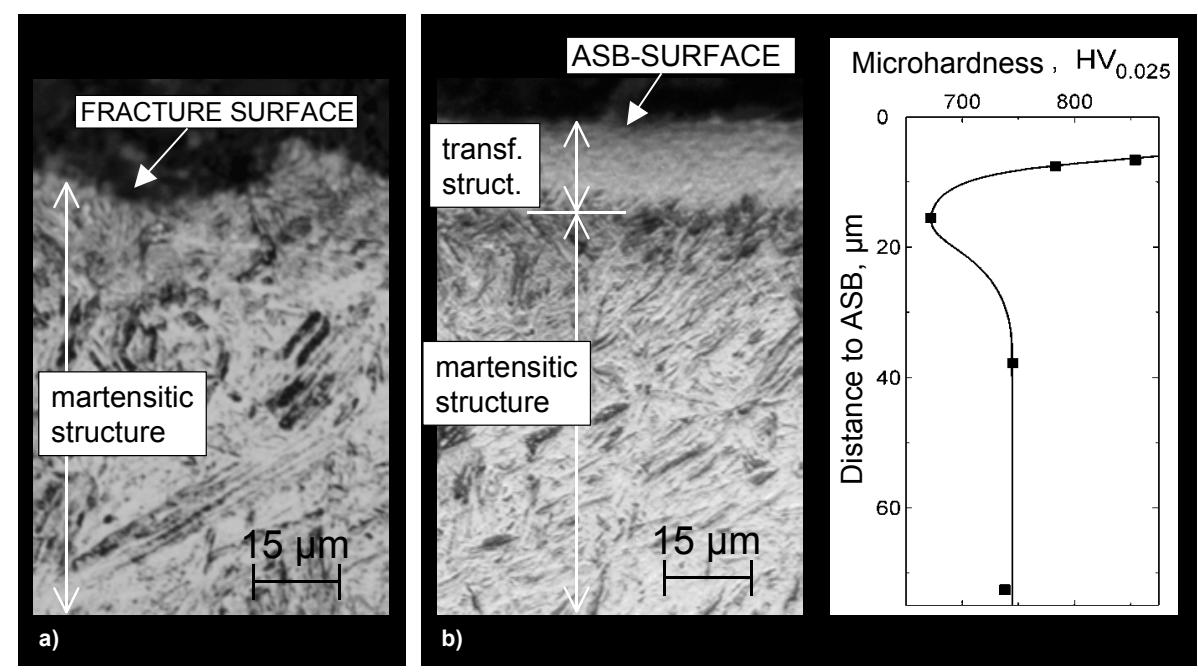

Figure 8: Transformation of the microstructure and increase in micro-hardness in the vicinity of an adiabatic shear band (b) compared to the behaviour near a fracture surface (a)

The above findings and conclusions are essential for establishing optimized conditions for separating material. The general trend that lower amounts of energy are required when the loading rate is increased does not hold anymore and is reversed when the failure mode changes from cracks to adiabatic shear bands.

\section{ACKNOWLEDGEMENT}

The research work has been supported by the Office of Naval Research under the technical direction of Dr. Yapa D.S. Rajapakse.

\section{REFERENCES}

1. Kalthoff, J.F. (1990) Applied Mechanics Review 43, 247

2. Kalthoff, J.F. (2000) International Journal of Fracture 101, 1

3. Bürgel, A. (2000). Dissertation, Ruhr-Universität Bochum, Germany.

4. Bürgel, A., Shin, H.S., Bergmannshoff, D. and Kalthoff, J.F. (1999). In: Proc. VII. Bilateral Czech/German Symposium: Significance of Hybrid Method for Assessment of Reliability and Durability in Engineering Sciences, 13.-15. April 1999 Liblice, Czech Republic, 11

5. Dally, J.W. and Sanford, R.J. (1987) Exp. Mech. 27, 381

6. Hiese, W. (1999). Dissertation, Ruhr-Universität Bochum, Germany.

7. Hiese, W. and Kalthoff, J.F. (1999) In: Mixed-Mode Crack Behavior, ASTM STP 1359, pp. 74-85, K.J. Miller and D.L. McDowell (Eds.). American Society for Testing and Materials, West Conshohocken, PA 